Meta

Journal des traducteurs

Translators' Journal

\title{
D’une langue en plus
}

\section{Liliane Ickowicz-Zolty}

Volume 27, numéro 1, mars 1982

Psychanalyse et traduction

URI : https://id.erudit.org/iderudit/004647ar

DOI : https://doi.org/10.7202/004647ar

Aller au sommaire du numéro

Éditeur(s)

Les Presses de l'Université de Montréal

ISSN

0026-0452 (imprimé)

1492-1421 (numérique)

Découvrir la revue

Citer cet article

Ickowicz-Zolty, L. (1982). D’une langue en plus. Meta, 27(1), 106-112.

https://doi.org/10.7202/004647ar

Ce document est protégé par la loi sur le droit d'auteur. L'utilisation des services d'Érudit (y compris la reproduction) est assujettie à sa politique d'utilisation que vous pouvez consulter en ligne.

https://apropos.erudit.org/fr/usagers/politique-dutilisation/
Cet article est diffusé et préservé par Érudit.

Érudit est un consortium interuniversitaire sans but lucratif composé de l’Université de Montréal, l'Université Laval et l'Université du Québec à Montréal. Il a pour mission la promotion et la valorisation de la recherche. https://www.erudit.org/fr/ 


\section{D'UNE LANGUE EN PLUS}

LILIANE ICKOWICZ-ZOLTY

C'est du côté de Freud que nous sommes allés interroger ce qu'il en est de la transmission, car si Freud nous a transmis la psychanalyse, c'est que ce dont il a lui-même hérité rendait possible ce passage à la psychanalyse et sa transmission.

Qu'est-ce qui s'est transmis à Freud?

Nous faisons nôtre, pour avancer l'hypothèse qui suivra, ce qu'écrit Lacan dans le Séminaire sur le Moi :

La question du sujet... concerne son histoire en tant qu'il la méconnaît et c'est là ce qu'il exprime bien malgré lui à travers toute sa conduite, pour autant qu'il cherche obscurément à la reconnaître. Sa vie est orientée par une problématique qui n'est pas celle de son vécu, mais celle de son destin à savoir qu'est-ce que son histoire signifie.

Ce qui fait effet de parole et d'écriture pour Freud est sa part méconnue. Or quoi de plus méconnu que le discours de la mère; non pas la langue que la mère parle, mais le discours qui la parle, qui lui est à elle-même méconnu, méconnaissance qui se transmet et vient marquer le sujet qui en devient luimême le porteur.

Le yiddish, dans sa fonction de méconnaissance pour Freud nous paraît pouvoir poser la question de savoir comment, de la transmission de cette méconnaissance, a pu se constituer un discours dans le rapport à une autre langue. C'est à ce point que nous sommes allés l'interroger et interroger par là-même notre propre méconnaissance.

Je souhaiterais succinctement faire état de notre rencontre avec cette langue dans quelques-uns de ses aspects qui parlent d'eux-mêmes et tels qu'ils ont également pu parler à Freud.

Je rappellerai que le yiddish occupe une position très particulière, c'est une langue qui emprunte ses signifiants aux autres langues.

Composé essentiellement de l'allemand de l'ancienne Lotharingie, il a été agrémenté au cours des déplacements : de polonais, de russe, d'apports hébreux et plus récemment de français et d'américain. L'emprunt se fait au niveau des mots mais dans une écriture différente : l'écriture hébraïque. Cela pose immédiatement la question de l'identification. À la différence des autres langues dans lesquelles circulent également des emprunts, on ne donne rien en retour, sinon la possibilité de faire apparaître une position de sujet. En empruntant à la fois à l'allemand et à l'hébreu les instruments de son élaboration, le yiddish s'inaugure du symbole de sa propre castration. Cette situation privilégiée le met en position de toujours devoir reproduire ce signifiant de son moment inaugural. 
Le yiddish est toujours «up to date», branché sur le sens, il emprunte à mesure les signifiants devenus nécessaires. On pourrait dire que du fait de ses emprunts, le yiddish opère une rupture dans les chaînes signifiantes des langues auxquelles il emprunte, et, privé de leurs associations qu'il brise, il doit produire dans sa structure même ces effets de signification. La structure a remplacé les chaines associatives. Les mots yiddish ne veulent pas d'histoire... et pas de passé. Ils sont à n'importe qui et la langue est prête à s'en séparer. Le yiddish doit faire apparaitre dans la trame même de son discours, ce qui est évacué dans l'histoire signifiante de ses emprunts. Il fonctionne alors comme interprète.

Le yiddish s'inaugure de cette rencontre signifiante, c'est ce qu'il transmet et qu'il ne cesse de répéter. Cette rencontre touche au réel, fonde la langue comme le rêve qui met en scène la rencontre du sujet avec le discours et ne peut en témoigner que par images. Le yiddish est une langue d'images et d'allégories.

Par son acte inaugural de brisure et en se constituant comme discours, le yiddish précipite les signifiants d'emprunt au sens chimique du terme c'est-à-dire produit un style parfaitement hétérogène aux éléments qui l'ont constitué mais sans lesquels il n'est rien.

L'histoire du yiddish est l'histoire de ce renversement, aussi absurde apparemment que l'est ce rêve que cite Freud dans la Traumdeutung :

«Un homme rêve qu'il va dans une gare où un train arrive. On rapproche alors le trottoir du train arrêté.» En yiddish, on prend les trottoirs en marche.

Toute rencontre de deux parleurs yiddish perpétue de cette manière ce renversement. La parole qui inaugure la rencontre inverse le message :

«Chouleym Aleykhem, Reb David.

- Aleykhem Chouleym, Reb Isaac, renvoie l'autre en écho.

Cette langue n'est pas différente des autres langues de par les effets qu'elle doit produire. Comme toute langue elle marque les impératifs, elle rend possible l'exploitation, elle a les mêmes incidences de sexe, les mêmes effets hystériques, elle opère comme langue constituée, les mêmes effets de dominance du savoir et surtout, elle permet de rêver, c'est-à-dire de supporter le réel, mais ce qui nous intéresse c'est son moment inaugural et la manière dont elle s'y prend.

Ce n'est bien sûr pas spécifique du yiddish de devoir produire, de par sa position de langue méconnue, voire interdite, les effets qu'elle supporte. On pourrait même dire que toute langue qui fonctionne à l'insu des langues dominantes amène à produire les effets de signifiant qu'elle véhicule, et que c'est cette langue oubliée qui organise la culture dans le rapport à cette autre langue dominante.

Chaque langue a une structure et une histoire différentes, et ce qu'elle vient produire de par sa spécificité se marque donc différemment.

C'est la spécificité de la langue yiddish, dans son moment inaugural, moment inaugural qui est véhiculé dans la langue qui a pu, à l'insu de Freud, révéler une structure métonymique, celle de l'inconscient.

Essayons d'en rendre compte! 
Reprenons cette histoire yiddish que Freud cite et qui a souvent été commentée :

Deux concurrents se rencontrent un jour dans un train :

- Où allez-vous Reb David?

- Je vais acheter du blé à Varsovie.

L'autre, entendant cela, sourit.

- Pourquoi souriez-vous Reb Isaac?

- Je ne comprends pas pourquoi vous me dites un mensonge.

- Un mensonge! quel mensonge? Je vous ai déjà beaucoup menti?

- Non, non, Reb David, je vous comprends très bien: vous me dites que vous allez à Varsovie chercher du blé afin que je croie que vous allez à Minsk chercher de la farine, mais je sais que vous allez vraiment à Varsovie pour du blé.

Cette histoire est bien une histoire supportée par la langue yiddish, car le yiddish sait que la vérité se donne d'elle-même malgré ce qui s'énonce et qu'elle est d'autant plus évidente qu'elle s'annonce par un mensonge ou une négation.

Ces deux concurrents se rencontrent dans un train, dans un non-lieu. En effet en yiddish on ne va jamais nulle part, et si les histoires se passent souvent dans un train c'est que pour arriver nulle part, il faut beaucoup voyager.

En yiddish on ne va pas à Varsovie, en tout cas pas directement.

Pour dire je vais à Varsovie le yiddish dit: «Ikh four kein Warsche», «je vais pas à Varsovie» et s'il n'y va pas, c'est bien qu'il y va.

Mais comment dire alors qu'il ne va pas à Varsovie? Le yiddish dira : «Ikh four nicht kein Warsche». Le yiddish nie alors qu'il ne va pas à Varsovie, donc il y va, donc il n'y va pas.

Voilà ce qu'ils se disent dans le transfert à non-Varsovie. Le premier sourit, car il est de plain-pied avec la règle du jeu que l'autre parleur yiddish organise. Il reconnait chez le premier cette complicité dans le mensonge qu'est le yiddish.

Cette histoire repose sur du semblant : l'un fait semblant de savoir ce que l'autre pourrait faire semblant d'ignorer.

Cette vérité qui ne peut se dire que dans la "dénégation » qu'organise ce transfert, c'est le yiddish comme lieu, transfert qu'elle organise pour survivre.

Cette structure de l'affirmation et de la négation, où la dénégation est utilisée pour poser une affirmation serait tout à fait incorrecte en allemand, langue dans laquelle oui c'est oui et non c'est non, au niveau du sens du moins.

Dirons-nous comme le yiddish: Freud ne parlait pas le yiddish? Freud hot nicht geredt kein yiddish. Il le parlait donc. C'est en tout cas notre hypothèse. Sourions comme notre voyageur sans pourtant vouloir rien comprendre à cet «aveu» de Freud dans Psychopathologie de la vie quotidienne:

On est étonné de constater, écrit-il, que le penchant à la vérité est beaucoup plus fort qu'on n'est porté à le croire. Il faut peut-être voir une conséquence de mes recherches psychanalytiques dans le fait que je suis devenu presque incapable de mentir. Toutes les fois que j'essaie de déformer un fait, je commets une erreur ou un autre acte manqué qui révèle mon manque de sincérité... 
Sourions... et sourds restons à cette cascade de dénégations subtiles comme le yiddish qui déploie une souveraine surdité. Le yiddish met en place la possibilité pour que chaque parleur soit un analyste possible pour l'autre. Il ne s'agit pas de se faire comprendre puisqu'en yiddish «être en prison»: in Tvisse sein, c'est être compris mais de se faire entendre.

Entendons cette histoire :

Deux parleurs se rencontrent dans un non-lieu. L'un demande à l'autre :

- Que faites-vous donc par ici?

- Boh... Boh...

- Votre affaire? ça marche bien?

- Boh...

- Votre femme que devient-elle?

- Boh... boh...

- Et vos petits-enfants?

- Boh... boh...

- Bon! alors bonne journée à vous!

- Bonne année à vous! Comme c'est bien d'avoir pu parler à un ami et soulager son cœur!

En yiddish c'est la rencontre qui est inaugurale. Cette inauguration est marquée par la langue qui renvoie à toute rencontre un : «bonne année!» inaugural, quel que soit le moment du jour ou de l'année.

En yiddish il ne s'agit pas de communiquer, ni de dialoguer, pas plus de se répondre ou de se comprendre; mais il s'agit de témoigner qu'on est vivant et que l'autre ne veut pas votre mort. Il y a là la reconnaissance d'un incompréhensible qui échappe au discours. Objet toujours inassimilable qui pousse à parler, à désirer et cependant toujours passer à côté. Le yiddish permet d'être "fous» de concert, à partir de l'impossibilité d'édifier un savoir qu'on est toujours en train d'inaugurer, donc un pouvoir. Le repérage de ce pouvoir du signifiant qui vient en permanence déséquilibrer l'édifice acquis ne fonde aucun pouvoir. Le yiddish $n$ 'est pas une institution et cela nous interroge que ce soit justement dans un rapport au yiddish qui n'est pas une institution que la psychanalyse soit née. Le yiddish n'est pas une langue rhétorique, c'est une langue rébus qui n'a rien à faire avec le concept. C'est un acte qui rend possible l'émergence de l'inconscient en venant briser ce qu'il en est du pouvoir des mots et. des mots du pouvoir. Les parleurs yiddish qui sont restés dans leur contexte culturel ne s'y trompent pas; ils sont réticents, voire hostiles à l'égard de l'élaboration psychanalytique et de son initiateur, considéré comme traître à la communauté. Cela n'est pas pour nous étonner. Le savoir qui a pu se constituer à partir de cette langue qui repose sur «pas de savoir» et qui viendrait lui faire retour est de nature si violemment hétérogène qu'il ne pourrait que pervertir son objet, forclore l'acte d'inauguration de la langue et y introduire une schize.

Avançons une autre observation: le yiddish fait opérer au sujet une place particulière dans le discours.

En yiddish on dit: "Ça me rêve»: es roulem zu mir. Ça m'a rêvé pour Freud qui est venu interroger ce «ça» qui nous rêve. Les songes sont comme les mensonges du yiddish et Freud s'est mis à leur écoute. 
Un autre effet du yiddish porte sur la nomination des parents. Dans cette langue les géniteurs sont directement introduits dans leur fonction symbolique. On ne dit pas : «Ma mère», ni «mon père» mais die Mame, der Tate : la mère, le père, et cette fonction étant assurée, on peut alors s'approcher du corps de la mère; un peu trop parfois, car nos parleurs étant souvent bilingues, cela peut créer quelques confusions.

Quant au père, der Vater s'il se décline, il ne se possède pas mais il circule :

\begin{tabular}{lllcc}
\hline ONCLE & PÈRE & MERE & TANTE & COUSIN \\
\hline $\begin{array}{l}\text { Der Vetter } \\
\text { Der Uncle }\end{array}$ & $\begin{array}{c}\text { Der Tate } \\
\text { Der Vater }\end{array}$ & $\begin{array}{c}\text { Die Mame } \\
\text { Digne supérieure }: \text { yiddish }\end{array}$ & $\begin{array}{c}\text { Die Mume } \\
\text { ligne inférieure : allemand }\end{array}$ & $\begin{array}{c}\text { Die Tante } \\
\text { Der Vetter }\end{array}$ \\
\hline
\end{tabular}

Der Vater allemand se retrouve en yiddish pour désigner l'oncle : der Vetter qui signifie cousin en allemand. La rencontre du yiddish et de l'allemand provoque une confusion dans les niveaux de génération: quant à la tante: Die Mume et la mère die Mame, c'est l'enfant qui tranche l'équivoque. En yiddish le cousin se dit: das Schwesterkind, c'est-à-dire l'enfant de la sœur, sous-entendu sœur ou belle-sœur du père ou de la mère car en yiddish le cousin ou la cousine se repère dans la filiation féminine. Cet enfant ainsi nommé, qui vient occuper dans la langue la place de l'enfant imaginaire n'est pas loin de révéler les positions incestueuses, et c'est parce qu'il vient ainsi s'y risquer, que le rappel à la fonction symbolique du père se doit d'être inébranlable.

Comment cette circulation particulière de la dénomination parentale en yiddish a-t-elle pu affecter Freud?

Choisissons quelques exemples :

Quel est l'oncle, interroge Freud dans la Traumdeutung qui n'a pas fait voler un enfant, le transportant à bras tendus et courant à travers la pièce, ou qui n'a pas joué à le laisser tomber en étendant brusquement les jambes alors qu'il le balançait sur ses genoux... les enfants poussent des cris de joie... des années après ils répéteront cela dans le rêve, mais ils oublieront les mains qui les ont portés de sorte qu'ils voleront et tomberont librement.

Cette invocation de l'oncle (der Vetter en yiddish) alors qu'il s'agit manifestement du père ne nous surprend pas. Il y a là glissement du registre de l'allemand au yiddish. Quant à cet oubli dans le rêve dont parle Freud, il nous étonne, car il nous l'a fait savoir et nous en a convaincu et c'est là le principe fondamental de son travail sur les rêves : le rêve n'oublie rien et surtout pas les mains qui ont en soutenant son corps d'enfant inscrit à tout jamais l'étreinte maternelle.

La confusion que le signifiant VATER-VETTER, père-oncle, provoque chez Freud au carrefour de deux langues, reconstitue l'équivoque qui existe dans la langue yiddish lorsque l'enfant imaginaire vient à forcer le questionnement. Questionnement que Freud cherche en même temps à annuler en renonçant pour un instant à son travail de conceptualisation.

À un autre endroit, dans Psychopathologie de la vie quotidienne à propos de l'analyse de ses «erreurs de mémoire» dont Freud dit qu'elles sont facile- 
ment explicables par l'analyse, il évoque une erreur qui figure dans la Science des rêves:

«J'affirme que Zeus a émasculé et renversé du trône son père Chronos. J'ai par erreur fait avancer cette horreur d'une génération: la mythologie grecque l'attribue à Chronos à l'égard de son père Ouranos.»

Équivoque donc d'une génération comme pour le yiddish. Il est intéressant de noter que cette «erreur" de Freud est d'ailleurs mentionnée comme douteuse, puisque d'après la version orphique du mythe, l'émasculation de Chronos fut bien l'œuvre de son fils Zeus. À propos de cette "erreur» toujours, Freud évoque ce conseil de son frère qui lui est resté en mémoire:

«En ce qui concerne ta conduite dans la vie me disait-il, il est une chose que tu ne dois pas oublier: «tu appartiens non à la deuxième, mais à la troisième génération à partir de celle de notre père. »

Je voudrais encore ajouter, et ce sera une manière de conclure, qu'en yiddish on n'apprend rien mais on s'apprend.

Pour dire «j'apprends » le yiddish dira Ikh lerne mir : «Je m'apprends. » Et là où l'allemand utilise deux mots : lehren, enseigner et lernen, apprendre, le yiddish n'en produit qu'un seul: lernen. En yiddish enseigner et apprendre (s'apprendre) c'est la même chose.

Peut-on alors apprendre ce que le yiddish enseigne? Peut-on enseigner voire transmettre ce que le yiddish apprend? Faut-il inaugurer un autre discours?

Le yiddish touche au cœur de son existence même à cette question de la transmission. Comment transmettre en effet alors qu'il n'y a pas d'enseignement qui ne soit en même temps ce que le sujet apprend de lui-même, à lui-même et que toute communication fonctionne que de n'être pas comprise de sorte à lui faire retour sous la forme dévoilée que nous avons essayé d'illustrer plus haut. C'est à cette gageure du yiddish que Freud a cherché à répondre en inaugurant, comme l'a fait le yiddish, un autre discours : celui sur l'inconscient.

Nous avons avancé que le yiddish fonctionne comme interprète des langues d'emprunt. C'est de cette position d'interprète soutenue par la langue yiddish que Freud a lui-même opéré dans le champ de la langue allemande et retrouvé une trame similaire, celle de l'inconscient, qu'est venu lui souffler le yiddish.

La communication qui va suivre, s'essaye à rendre compte de ce moment précis de la rencontre de Freud avec l'inconscient, à ce carrefour privilégié du yiddish et de l'allemand.

Cette méconnaissance du yiddish par Freud dont nous avons dit qu'elle l'avait guidé vers la psychanalyse, est venue s'inscrire dans ses écrits mêmes où il en a maintenu le statut qui a rendu possible son écriture. Cette méconnaissance a porté sur le sort fait par Freud à ses écrits dits "pré-analytiques » qu'il a écartés de ses Gesammelte Werke. Le pré-analytique est-il un discours sur la langue yiddish, discours métaphorique dans le choix des objets étudiés et dans la manière dont Freud en témoigne?

La troisième de nos communications cherche à interroger cette circulation de la méconnaissance dans la généalogie des préoccupations de Freud qui l'a poussé à cette nécessité de la découverte psychanalytique. 
Et, restera ouverte cette question qui nous vient aujourd'hui :

- le yiddish, métaphore de tout discours inaugural, discours d'un sujet, s'est-il transmis avec la psychanalyse comme son enfant illégitime?

Le yiddish, pensons-nous, c'est le fantasme de Freud qui soutient son désir d'être analyste, fantasme qui a affaire avec l'inceste, avec la transgression de la loi.

Cet enfant illégitime, comment s'autoriser sans le reconnaître? comment s'habiliter sans le méconnaître? 